Celine Wegner*, Paul Schlett, Julian Höth, Thomas Buckert, Thomas Klotzbücher, Ulrich G. Hofmann, and Thilo B. Krüger

\title{
Neurophotonic Scanning System - Towards Automatic Infrared Neurostimulation
}

\begin{abstract}
Intraoperative neuromonitoring is without doubt important for all surgical interventions, where nerve structures are at risk. Mapping techniques for the identification of motor nerves and cortex are available. They rely on manual identification using an electrical stimulation probe. A landmark-based visualization of the results is currently not possible. To overcome these limitations, we are developing a system for automatic stimulation and display of functional tissue.

Infrared neurostimulation (INS) was proposed to be a feasible alternative to electrical stimulation of nerves. It provides contactless and artifact-free activation of nerves. For our preclinical experiments we used an infrared diode laser system with a wavelength of $1470 \mathrm{~nm}$. For automatic screening, we developed a scanning system and suitable scan-algorithms in order to provide optimal scanning parameters. Recording of compound muscle action potentials (CMAP) was performed with a differential amplifier and appropriate neurophysiologic software.

Subunits of the system were combined via multiple interfaces. A control-software merges all relevant functions of the individual parts and parallel use. Marking of tissue was realized with a red pilot laser deflected by the same scanning system.

With this work we could show, that a system for automatic laser deflection with parallel neurophysiologic recording and subsequent highlighting of irradiated tissue is possible. This system can serve as a tool for further systematic investigations in the field of INS.
\end{abstract}

\footnotetext{
*Corresponding author: Celine Wegner: inomed Medizintechnik $\mathrm{GmbH}$, Im Hausgrün 29, 79312 Emmendingen, Germany, c.wegner@inomed.com

Paul Schlett, Ulrich G. Hofmann: Section for Neuroelectronic Systems, Uniklinik Freiburg, Freiburg, Germany Julian Höth, Thomas Klotzbücher: Fraunhofer-Institut für Mikrotechnik und Mikrosysteme IMM, Mainz, Germany Thomas Buckert: ARGES GmbH, Wackersdorf, Germany Thilo B. Krüger: inomed Medizintechnik $\mathrm{GmbH}$ Emmendingen, Germany
}

Keywords: Intraoperative Neuromonitoring, Surgical Mapping, Neurostimulation, Infrared Optical Stimulation, Laser Stimulation

https://doi.org/10.1515/cdbme-2020-3069

\section{Introduction}

Intraoperative neuromonitoring is gaining in importance for all surgical interventions, where nerve structures are at risk of damage. Mapping techniques for the identification of nervous tissue responsible for motor skills, so called functional tissue, are available and rely on manual identification using an electrical stimulation probe [1]. They are widely applied for example during thyroid surgery to identify the recurrent laryngeal nerve or in the neurosurgical field in order to map functional tissue in the brain. They enable the surgeon to stimulate during the resection of a tumor even without changing tools and serve as a control of distance to, for example, the corticospinal tract [2]. A similar method exists for thyroid surgery, where continuous monitoring is performed using a cuff electrode on the vagal nerve [3].

However, a visualization of tissue classified as functional by mapping is currently not possible. Instead, the surgeon must remember the relevant spots by himself, constantly repeating the mapping process, or annotating sites with markers, which often shift, due to irrigation or the resection process.

In order to overcome these limitations, we are developing a system for automatic stimulation and marking of functional tissue, which is based on infrared neurostimulation (INS) by a diode laser.

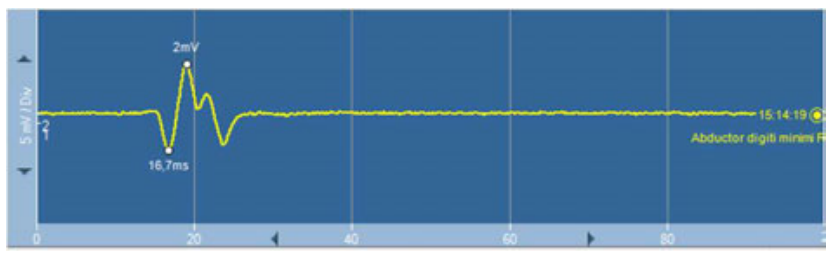

Figure 1: Peak detection in CMAP recording. 


\section{Methods}

\subsection{Infrared Neurostimulation}

INS has been proposed to be a possible alternative to electrical stimulation for activation of peripheral nerves [4, 5]. It provides the advantage of contactless and artifact-free stimulation.

For our INS setup, we use an infrared diode laser (1470 nm, $12 \mathrm{~W}$, DILAS Diodenlaser GmbH, Mainz, Germany), which has been shown to successfully elicit compound muscle action potentials (CMAP) after optical sciatic nerve stimulation in rats [6].

In order to ensure flexible stimulation locations, we use a custom-made scanning system (ARGES GmbH, Wackersdorf, Germany) to deflect the laser beam. This enables automatic screening of areas, such as an operation site. The scanning system includes two mirrors and thereby enables the laser spot to hit the surface of a two-dimensional operation site at discretionary locations. Additionally, the scanning system is designed to achieve preferred focus spot values with the chosen diode laser. The scanning system is driven by an external controller, which also enables operation of the infrared diode laser system.

\subsection{Electrophysiological Recording}

Successful stimulation of functional tissue for motor skills results in muscle movement or other downstream evoked potentials. Muscle movements can be recorded as CMAP. In our system, this is performed with a differential neurophysiologic amplifier and appropriate software (ISIS Headbox and NeuroExplorer, inomed Medizintechnik GmbH, Emmendingen, Germany). The software enables automatic detection of peaks and calculation of the amplitude (Figure 1).

\section{Results}

\subsection{Scanning Unit}

The scanning unit is a compact system with a complex optical structure in order to fulfill the requirements of infrared beam steering (Figure 2). The laser beam is coupled to the scanner by a fiber with a core diameter of $200 \mu \mathrm{m}$. Simulations show that the achievable final spot size has a diameter of $1,000 \mu \mathrm{m}$ at a working distance of $200 \mathrm{~mm}$ and a field size of

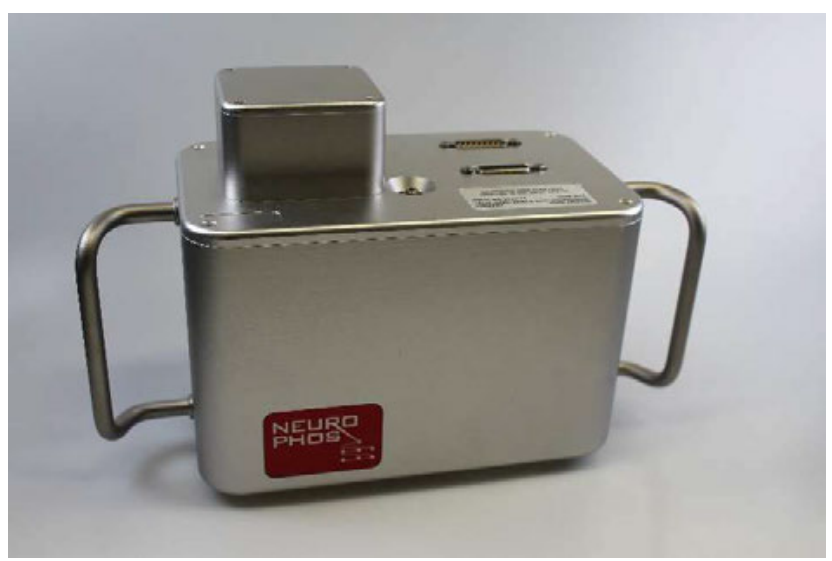

Figure 2: Scanning unit for the automatic laser deflection.

$30 \mathrm{~mm} \times 30 \mathrm{~mm}$. By exchanging one lens, the spot size can be minimized to a diameter of $500 \mu \mathrm{m}$ at a working distance of $100 \mathrm{~mm}$ and a field size of $18 \mathrm{~mm}$ x $18 \mathrm{~mm}$. Two integrated laser pointers indicate the focal plane. The scanner is prepared for use in the operating room, since a sterile cover can be applied. All electronics is integrated in an external controller to avoid heating of the scan head itself.

\subsection{Interfaces}

The single parts of the system are combined by using multiple interfaces. The customized controller operates the laser and scanner by a proprietary interface (ARGES GmbH, Wackersdorf, Germany).

The utilized neurophysiologic software offers streaming of recorded or meta data, such as peak information, via google Remote Procedure Calls (gRPC) network.

A control software merges all relevant functions of the individual parts and enables the operation. The interaction of all components is shown in Figure 3.

\subsection{Automatic irradiation}

The scanning unit allows fast and flexible movement of the laser spot to arbitrary locations in the situs. However, the minimal scanning time is limited by the physiological system and the expected latency of the CMAP. Therefore, a simple line-by-line scanning procedure of the complete situs would take too long to be applicable in a surgical setting. Instead, suitable scanning algorithms are developed and tested, in which upcoming stimulation spots are dependent on the stimulation result of the previously scanned spots. 


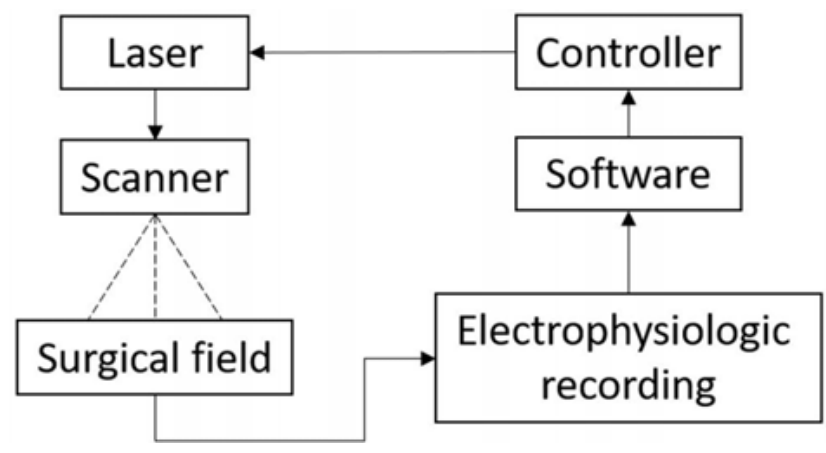

Figure 3: Concept of the combined system for laser scanning and electrophysiologic recording.

\subsection{Marking of functional tissue}

Marking of functional tissue is realized via a pilot laser, deflected by the scanning system. In this way the pilot laser is moved over the situs and irradiated tissue can be highlighted directly on the tissue.

\subsection{Demonstration of system}

Demonstration of the combination of the single parts of the system is performed in a laboratory setup. A nerve is simulated by printed circuit board tracks covered by paper tissue with water solvent. The tracks are connected to the CMAP recording unit. By irradiating these tracks with the laser, an artifact resembling a CMAP can be produced and automatically interpreted by the system. Negative results are produced if the tracks are not hit by the laser focus. After a scanning process the artificial nerve can be highlighted via the pilot laser by sequentially following the points with positive CMAP answers.

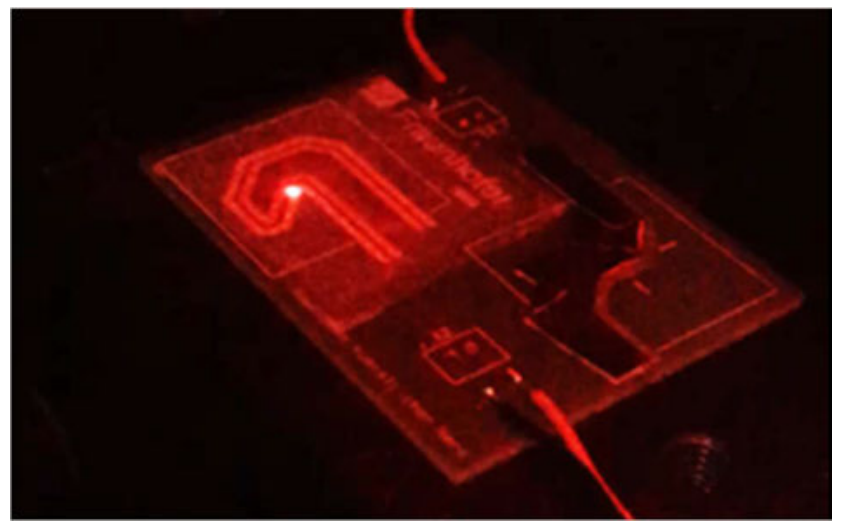

Figure 4: Artificial nerve with pilot laser spot

\section{Discussion}

Within this work we demonstrated, that the combination of laser scanning with a neurophysiologic system is possible. Via different interfaces the single parts could be fused into a demonstrator system, featuring automatic laser deflection, interpretation of neurophysiologic signals, and display. General technical functionality was shown in technical laboratory setup.

While technical feasibility of an automatic system for irradiation and marking of tissue is demonstrated, biophysical aspects still have to be explored and discussed. The underlying physiological efficacy of INS has not been finally described yet. Energy absorption by water and a resulting increase in tissue temperature has been proposed as one possible mechanism for nerve depolarization after laser irradiation [7, $8]$.

Our group performed INS studies on rats' sciatic nerves with the described laser and a fixed lens setup, which serves as the basis for the system described here [9]. Here, the sciatic nerve was irradiated with laser pulses focused to a spot of $450 \mu \mathrm{m}$ in diameter under EMG acquisition of multiple leg muscles simultaneous to high-speed thermal monitoring of the nerve surface. Like other groups investigating INS [4, 5], we had a high rate of successful nerve activation by infrared irradiation. Pulse energies above 7 to $8 \mathrm{~mJ}$ caused reliable motor unit activation, that evoked CMAP and visible muscle twitches. Successful nerve stimulation was observed in all 74 animals, with generally increasing CMAP amplitudes at increasing pulse energies. Furthermore, the results with the sophisticated technical setup with the fixed lens system show that nerve heating is highly dependent on the laser parameters such as pulse width, pulse energy, and pulse frequency [9]. Stimulation with a pulse frequency larger than $2 \mathrm{~Hz}$ leads to cumulated nerve heating, which can shortly exceed $43^{\circ} \mathrm{C}$. However, pulse energy threshold and CMAP amplitude was highly depending on the stimulated location on the nerve surface [9], which most likely originates from the inhomogeneous distribution of motor axons in peripheral nerves [10]. The high spatial dependency of nerve activation observed during translation of the laser spot across the nerve surface demonstrates the high resolution of the system to selectively activate axons or axon groups. These findings confirmed the concept of infrared sensitive regions and INS as a local and selective method for nerve activation.

Further investigations on the basic concepts of INS at one point must lead to a definition of save stimulation parameters. This aspect of laser stimulation is critical but challenging, mainly due to a broad variety of setups, parameters and definitions. Most setups described in publications are based on 
manual positioning of a laser fiber close to the exposed nerve. Our setup, however, is based on contactless irradiation with a defined working distance. The two integrated laser pointers for indicating the focal plane ensure working in an optimal distance and with stable optical parameters. In this way our system provides comparable optical parameters in different experiments and thereby comparable results.

INS has been proposed to be feasible for peripheral nerve stimulation. Target nerves are the sciatic or cavernous nerves of rats $[4,9,11]$, the auditory and facial nerve of gerbils [8, 12], or the avian embryonic heart [13]. However, for neurosurgical applications INS of the cortex and the excitability of pyramidal cells by laser irradiation must be further investigated.

Within this work we have developed and presented a novel tool for the investigation of laser stimulation, which enables automatic direction of the laser beam with parallel neurophysiologic recording and marking of tissue. This system can serve as a basis for further detailed and systematic research in the field of INS.

\section{Author Statement}

Research funding: This work was funded by the Federal Ministry of Education and Research (BMBF) in the NeuroPhos Project (Grant number 13GW0155A).

Conflict of interest: Celine Wegner and Thilo B. Krueger are full-time employees of inomed Medizintechnik $\mathrm{GmbH}$. Thomas Buckert is a full-time employee of ARGES GmbH. Ethical approval: The research related to animals complied with all the relevant national regulations and institutional policies for the care of the used animal.

\section{References}

[1] A. Szelényi et al., "Intraoperative electrical stimulation in awake craniotomy: methodological aspects of current practice," Neurosurg. Focus, vol. 28, no. 2, p. E7, 2010.

[2] A. Raabe, J. Beck, P. Schucht, and K. Seidel, "Continuous dynamic mapping of the corticospinal tract during surgery of motor eloquent brain tumors: evaluation of a new method: Clinical article," J. Neurosurg., vol. 120, no. 5, pp. 1015-1024, May 2014, doi: 10.3171/2014.1.JNS13909.

[3] F. Angeletti, P. B. Musholt, and T. J. Musholt, "Continuous Intraoperative Neuromonitoring in Thyroid Surgery," Surg Technol Int, vol. 27, pp. 79-85, 2015.
[4] J. Wells, C. Kao, E. D. Jansen, P. Konrad, and A. Mahadevan-Jansen, "Application of infrared light for in vivo neural stimulation," J. Biomed. Opt., vol. 10, no. 6, p. 064003, 2005, doi: 10.1117/1.2121772.

[5] J. M. Cayce et al., "Infrared neural stimulation of human spinal nerve roots in vivo," Neurophotonics, vol. 2, no. 1, p. 015007, Feb. 2015, doi: 10.1117/1.NPh.2.1.015007.

[6] P. Schlett, C. Wegner, T. Krueger, T. Buckert, T. Klotzbuecher, and U. G. Hofmann, "Experimental Setup for the Systematic Investigation of Infrared Neural Stimulation (INS)," in World Congress on Medical Physics and Biomedical Engineering 2018, vol. 68/3, L. Lhotska, L. Sukupova, I. Lacković, and G. S. Ibbott, Eds. Singapore: Springer Singapore, 2019, pp. 77-81.

[7] J. Wells et al., "Biophysical Mechanisms of Transient Optical Stimulation of Peripheral Nerve," Biophys. J., vol. 93, no. 7, pp. 2567-2580, Oct. 2007, doi: 10.1529/biophysj.107.104786.

[8] P. D. Littlefield, I. Vujanovic, J. Mundi, A. I. Matic, and C.$P$. Richter, "Laser stimulation of single auditory nerve fibers," The Laryngoscope, vol. 120, no. 10, pp. 20712082, Sep. 2010, doi: 10.1002/lary.21102.

[9] P. Schlett, C. Wegner, T. B. Krueger, and U. G. Hofmann, "Towards Safe Infrared Nerve Stimulation: A Systematic Experimental Approach," in 2019 41st Annual International Conference of the IEEE Engineering in Medicine and Biology Society (EMBC), Berlin, Germany, Jul. 2019, pp. 5909-5912, doi: 10.1109/EMBC.2019.8857257.

[10] J. Badia, A. Pascual-Font, M. Vivó, E. Udina, and X. Navarro, "Topographical distribution of motor fascicles in the sciatic-tibial nerve of the rat," Muscle Nerve, vol. 42, no. 2, pp. 192-201, Aug. 2010, doi: 10.1002/mus.21652.

[11] S. Tozburun, C. D. Stahl, T. C. Hutchens, G. A. Lagoda, A. L. Burnett, and N. M. Fried, "Continuous-wave Infrared Subsurface Optical Stimulation of the Rat Prostate Cavernous Nerves Using a 1490-nm Diode Laser," Urology, vol. 82, no. 4, pp. 969-973, Oct. 2013, doi: 10.1016/j.urology.2013.06.031.

[12] I. U. Teudt, A. E. Nevel, A. D. Izzo, J. T. Walsh, and C.-P. Richter, "Optical Stimulation of the Facial Nerve: A New Monitoring Technique?:," The Laryngoscope, vol. 117, no. 9, pp. 1641-1647, Sep. 2007, doi: 10.1097/MLG.0b013e318074ec00.

[13] M. W. Jenkins et al., "Optical pacing of the embryonic heart," Nat. Photonics, vol. 4, no. 9, pp. 623-626, Sep. 2010, doi: 10.1038/nphoton.2010.166. 